\title{
Mesurer la sécrétion nectarifère : exemple d'une lignée hybride F1 et de son parent mâle stérile chez le colza d'hiver (Brassica napus L.)
}

\author{
Stan Chabert ${ }^{1,2, *}$, Taïna Lemoine ${ }^{2}$, Louna Fronteau ${ }^{2}$ et Bernard E. Vaissière ${ }^{2,3}$ \\ 1 Association nationale des agriculteurs multiplicateurs de semences oléagineuses, Ferme expérimentale, 2485 route des Pécolets, \\ 26800 Étoile-sur-Rhône, France \\ ${ }^{2}$ Institut national de la recherche agronomique, UR406 Abeilles et Environnement, 228 route de l'aérodrome, site Agroparc, \\ domaine Saint-Paul, CS 40509, 84914 Avignon cedex 9, France \\ ${ }^{3}$ UMT Protection des Abeilles dans l'Environnement, 228 route de l'Aérodrome, site Agroparc, domaine Saint-Paul, CS 40509, \\ 84914 Avignon cedex 9, France
}

Reçu le 5 octobre 2017 - Accepté le 7 novembre 2017

\begin{abstract}
Résumé - Au cours de l'histoire évolutive des plantes à fleurs, l'apparition des nectaires floraux a permis de substituer le pollen par du nectar pour attirer les animaux pollinisateurs, permettant de diminuer les coûts de la pollinisation animale liés à la consommation du pollen. Dans les productions de semence hybride des cultures entomophiles, connaître le niveau de sécrétion nectarifère des lignées en présence, mâle fertile (MF) et mâle stérile (MS), est important si l'on souhaite maximiser les transferts de pollen entre les deux. Dans cet article, nous faisons tout d'abord une revue des méthodes qui existent pour mesurer la sécrétion nectarifère, puis retenons celle qui mesure un taux de sécrétion brut, qui permet d'exprimer une vitesse de sécrétion, pour l'utiliser sur deux lignées de colza d'hiver (Brassica napus L.), la variété hybride F1 Exocet MF et son parent MS. Nous montrons que la sécrétion nectarifère du colza est constante sur un intervalle de temps de 6-8 heures durant les heures du jour, que cette sécrétion admet une température optimale se situant entre $20^{\circ} \mathrm{C}$ et $30^{\circ} \mathrm{C}$, et qu'elle est autour de deux fois moindre chez le parent MS par rapport à la lignée $\mathrm{F} 1$. Ces résultats permettent de proposer une méthode de mesure rigoureuse pour comparer la sécrétion nectarifère entre lignées ou variétés. Nous concluons sur les principales autres variables dont il faudrait tenir compte pour notamment pouvoir estimer la quantité totale de nectar sécrétée par une surface donnée de culture.
\end{abstract}

Mots clés : méthode / sécrétion nectarifère / colza / semence hybride / température

\begin{abstract}
Measuring nectar secretion: the example of an F1 hybrid and its male sterile parent in winter oilseed rape (Brassica napus L.). During the evolutionary history of flowering plants, the appearance of floral nectaries allowed the replacement of pollen by nectar to attract pollinators, allowing lower costs involved in animal pollination by reducing the consumption of pollen. In the hybrid seed productions of entomophilous crops, knowing the levels of nectar secretion of the different lines, the male fertile (MF) and the male sterile (MS) ones, is important to maximize pollen transfers between them. In this study, we start with a review of current methods used to measure nectar secretion, and choose the one that provides a gross secretion rate in order to use it on two winter oilseed rape (Brassica napus L.) lines, the hybrid F1 'Exocet' and its MS parent. We show that oilseed rape has a gross nectar secretion rate that is constant over a period of 6-8 hours during daylight hours, that it has a thermal optimum included between $20^{\circ} \mathrm{C}$ and $30^{\circ} \mathrm{C}$, and that the parental MS line secretes about half as much as the hybrid $\mathrm{F} 1$ one. These results enable us to propose a rigorous method to compare nectar secretions between lines and varieties. We conclude with the main other variables that should be taken into account to estimate the total amount of nectar produced by a given area of crop.
\end{abstract}

Keywords: method / nectar secretion / oilseed rape / hybrid seed / temperature

\footnotetext{
*Correspondance : stanchabert@gmail.com
} 


\section{Introduction}

\subsection{Origine évolutive des nectaires floraux}

Les premières plantes à fleurs dotées de nectaires floraux sont apparues au cours du Crétacéil y a environ 100 millions d'années (Friis et al., 2011), période qui correspond également à celle de l'apparition et de la diversification des abeilles (Poinar et Danforth, 2006; Michez et al., 2012; Branstetter et al., 2017), principal groupe d'animaux pollinisateurs de ces plantes, qui laisse supposer une co-radiation évolutive passée entre ces deux groupes (Michez et al., 2012; Cappellari et al., 2013).

Un grand nombre de plantes à fleurs actuelles sécrètent du nectar (Percival, 1961 ; Bernardello, 2007), mais ceci n'est pas un caractère propre à l'ensemble des Angiospermes: la très grande diversité de formes, structures et positions florales des nectaires laisse supposer de multiples événements d'apparitions indépendantes et de disparitions de ce caractère au cours de l'évolution au sein des différentes lignées évolutives (Bernardello, 2007). Les nectaires ont par exemple disparu chez les fleurs du genre Solanum (exemple : la tomate), où seul le pollen attire les insectes qui les visitent, alors qu'ils sont présents dans la plupart des autres genres de la famille des Solanaceae. De même, les Poaceae ont perdu leurs nectaires ancestraux, parallèlement à un retour à une pollinisation abiotique stricte.

\subsection{La sécrétion nectarifère florale est-elle forcément synonyme d'entomophilie?}

Le nectar a ainsi permis de se substituer au pollen dans l'attraction des insectes pollinisateurs chez un certain nombre de plantes à fleurs, permettant de diminuer les coûts de la pollinisation entomophile liée à la consommation de pollen par ces insectes (Takhtajan, 1980). La sécrétion de nectar est donc la plupart du temps associée à un mode de pollinisation entomophile, tout au moins partiel, mais ceci n'est pas systématique: un certain nombre de fleurs de l'archipel Juan Fernández au Chili sont par exemple exclusivement anémophiles, du fait de l'absence de visites d'insectes pollinisateurs, rares sur ces îles, alors qu'elles sécrètent pourtant du nectar (Anderson et al., 2000a, b, 2001; Bernardello et al., 2000, 2001). Les auteurs font l'hypothèse que ces espèces ont perdu le caractère entomophile de leurs ancêtres continentaux, sans avoir encore perdu le caractère de sécrétion de nectar.

Ceci est d'autant plus vrai en agriculture, où la sélection humaine de certaines variétés a pu conduire à la perte du caractère entomophile, avec toutefois un maintien de la sécrétion de nectar (Vaissière, 1991). Ce n'est donc pas parce que les fleurs d'une culture produisent du nectar, que les insectes qui visitent cette culture améliorent forcément son niveau de pollinisation, et par extension les rendements, de cette culture.

\subsection{La particularité des productions de semence hybride}

Ce n'est en revanche pas le cas des cultures entomophiles en production de semence hybride qui alternent une lignée mâle fertile (MF) avec une lignée mâle stérile (MS), où la lignée MS est celle sur laquelle la semence est récoltée et qui ne produit pas de pollen : l'autopollinisation y est impossible, de même que l'entomophilie s'il n'y a pas de sécrétion nectarifère. Pierre et Renard (2010) ont évalué le niveau moyen de dépendance à l'entomophilie de la production de semence hybride chez le colza à $90 \%$. La sécrétion nectarifère doit donc y être suffisamment importante pour attirer les insectes pollinisateurs, notamment par rapport à la production de nectar des autres plantes nectarifères présentes potentiellement dans l'environnement de la culture et qui peuvent entrainer une dilution importante des insectes pollinisateurs (Garibaldi et al., 2017). Enfin, si l'on souhaite maximiser les transferts de pollen de la lignée MF vers la lignée MS, Pierre et al. (1999) ont suggéré que la production de nectar ne devait pas être trop disproportionnée entre les deux lignées. Dès lors, il est fondamental de savoir mesurer correctement la sécrétion nectarifère d'une espèce, d'une variété ou d'une lignée données.

\subsection{Mesurer la sécrétion nectarifère}

Il existe différentes méthodes pour mesurer la quantité de nectar disponible dans une fleur. Ces différences portent sur le matériel utilisé, ou sur le traitement de la fleur préalablement à la mesure. Concernant le matériel, la méthode la plus couramment choisie est celle utilisant des microcapillaires, étant simple, rapide et fiable (McKenna et Thomson, 1988; Mesquida et al., 1988). Une méthode de récolte de nectar par centrifugation des fleurs a été mise au point pour obtenir rapidement des volumes de nectar suffisamment importants pour permettre l'analyse des constituants du nectar par chromatographie (Bosi, 1973). Mais cette méthode fournit un nectar artificiellement dilué et elle n'est pas exempte de modifier la composition chimique du nectar par lésion des tissus de la fleur (Mesquida et al., 1988). Des méthodes de lavage et de rinçage des fleurs avec de l'eau distillée ont été proposées pour échantillonner le nectar des fleurs contenant des petits volumes de nectar inférieurs à $1 \mu \mathrm{L}$ qui sont difficiles à échantillonner (Mallick, 2000; Morrant et al., 2009). Ces méthodes permettent d'échantillonner plus de nectar que celle utilisant les microcapillaires dans ce type de fleur, mais la question de savoir laquelle de ces méthodes reflète le plus fidèlement la quantité de nectar pouvant être effectivement extraite par les insectes floricoles lors de leur visite reste posée (Petit et al., 2011).

Il existe ensuite différents traitements de la fleur, préalablement à la mesure de la quantité de nectar, que nous examinons dans les paragraphes suivants.

\subsubsection{Nectar résiduel}

Une première méthode consiste à mesurer le «nectar résiduel» (nectar standing crop), c'est-à-dire la quantité de nectar disponible dans une fleur offerte au libre butinage des insectes (Kearns et Inouye, 1993; Corbet, 2003). Cette mesure, qui a l'intérêt de mettre en évidence la quantité moyenne de nectar réellement proposée par les fleurs aux insectes à un moment donné, souffre d'une très grande variabilité entre fleurs due au hasard de la durée écoulée entre la mesure et la dernière visite d'insecte, et ne permet pas de mettre en évidence les paramètres du processus de sécrétion en lui-même. 


\subsubsection{Taux de sécrétion apparent}

Une deuxième méthode, la plus classiquement utilisée, consiste à mesurer le taux de sécrétion apparent. L'échantillonnage s'effectue sur des fleurs ensachées sous tulle au moins 24 heures avant la mesure afin d'empêcher le butinage des fleurs par les insectes (Corbet, 2003). Cette méthode demeure pour le moment la méthode de référence pour estimer la quantité de nectar sécrétée par les plantes (exemple: Baude et al., 2016). Mais cette méthode considère la sécrétion de nectar comme un processus statique. Or, il a été montré que de nombreuses plantes, dont le colza, sécrètent du nectar en continu (Cruden et al., 1983; Pacini et Nepi, 2007), et de surcroît pour certaines d'entre elles, dont le colza encore, un processus de réabsorption se produit également en continu, de façon concomitante au processus de sécrétion (synthèse dans Nepi et Stpiczyńska, 2008). Ce processus de réabsorption est inhibé chez un certain nombre d'espèces lorsque le nectar est régulièrement retiré de la fleur (Luo et al., 2014), et ce notamment chez le colza (Búrquez et Corbet, 1991). Une fleur régulièrement butinée par un insecte produit donc plus de nectar en cumulé qu'une fleur non butinée. Cette méthode peut donc conduire à des estimations erronées de la quantité totale de nectar sécrétée par une fleur accessible aux insectes.

\subsubsection{Taux de sécrétion brut}

Une troisième méthode, qui mesure le taux de sécrétion brut, consiste à retirer totalement le nectar d'une fleur à un instant donné, à l'ensacher sous tulle, puis à mesurer la quantité de nectar produite après un certain intervalle de temps, à déterminer (Corbet, 2003). Ceci permet d'exprimer une vitesse de sécrétion nectarifère tout en s'affranchissant des processus de réabsorption, processus qui sont inhibés lorsque la fleur est régulièrement butinée. C'est donc cette méthode qui se révèle être la plus rigoureuse pour estimer des quantités de nectar sécrétées par des plantes butinées. Cette mesure est parfois appelée confusément taux de sécrétion net (exemple: Galetto et Bernardello, 2004).

\subsubsection{Convertir le volume en masse de sucres}

Enfin un certain nombre d'études ne mesurent que le volume de nectar sécrété. Or, il a été montré que la concentration du nectar admet une très grande variabilité, due principalement aux variations d'humidité relative atmosphérique (Pacini et Nepi, 2007). Il est donc préférable, dans la mesure du possible, de mesurer simultanément le volume et la concentration du nectar sécrété par une fleur, puis d'exprimer la quantité de nectar sécrétée en masse de sucres totaux dissous (STD) par l'intermédiaire d'une formule combinant ces deux variables. Deux formules ont été proposées à cet effet, et elles donnent des résultats quasiment identiques (Cruden et Hermann, 1983 ; Prys-Jones et Corbet, 2011).

\subsection{Les lignées MS sont-elles aussi nectarifères que les lignées MF?}

D'après le ressenti des apiculteurs, les rendements en miels des colonies d'abeilles mellifères sont moindres lorsqu'elles sont apportées sur des cultures de production de semence hybride. Une première étude a en effet pour l'instant montré

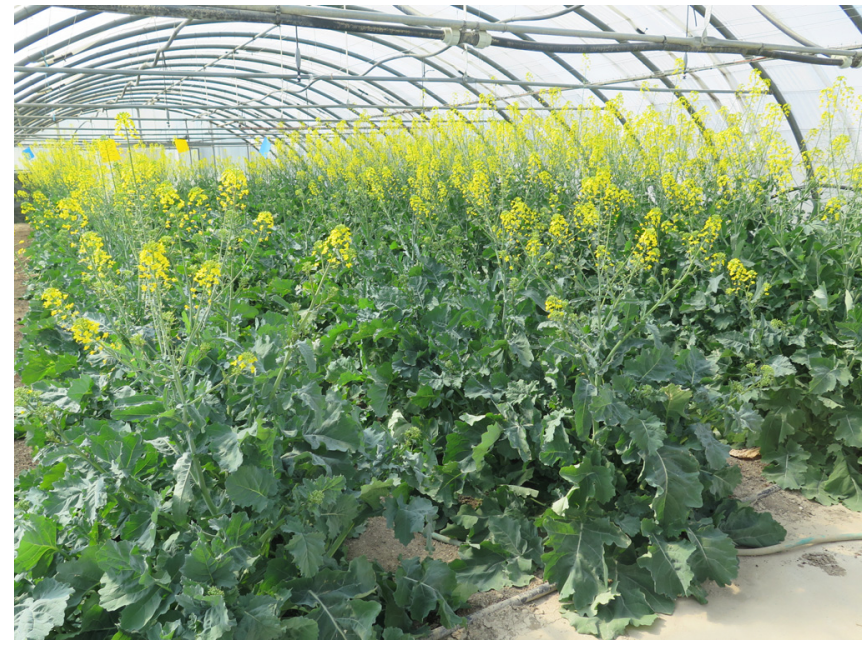

Fig. 1. Tunnel insectproof abritant les deux lignées de colza mâle stérile (les deux rangs de gauche) et F1 Exocet (les deux rangs de droite) en milieu de floraison. (C) Stan Chabert / Inra.

que trois lignées MS de colza produisaient de 16 à $70 \%$ de nectar en volume de moins que leurs homologues isogéniques MF (Pierre et al., 1999). Mais ces auteurs n'ont pas mesuré la concentration en STD de leurs échantillons de nectar pour connaître les quantités de STD effectivement produites. Ils ont par ailleurs mesuré le taux de sécrétion apparent, méthode qui souffre des biais évoqués précédemment.

La température joue enfin un rôle majeur sur la sécrétion nectarifère, bien que ce rôle n'ait été encore que relativement peu étudié (Pacini et Nepi, 2007; Petanidou, 2007).

Nous avons donc mesuré le taux de sécrétion brut de nectar au premier jour d'anthèse de fleurs de colza d'hiver d'une lignée hybride F1, mâle fertile, et de son parent MS afin de tester s'il y a une différence de vitesse de sécrétion brute entre les deux types de lignées. Nous avons réalisé ces mesures durant les heures de jour sur une gamme d'intervalles de temps compris entre le prélèvement initial et la mesure s'échelonnant de 15 minutes à 8 heures, ceci pour tester si (i) la sécrétion nectarifère reprend rapidement après un premier prélèvement et si (ii) cette sécrétion est constante tout au long des heures du jour après un premier prélèvement. Nous avons également testé l'effet de la température en répétant ces mesures sous tunnel dans différentes conditions de température ambiante durant les mois d'avril à mai. Enfin nous avons choisi la méthode utilisant des microcapillaires, adaptée pour le colza (Mesquida et al., 1988).

\section{Matériel et méthodes}

Les relevés ont été réalisés au printemps 2017 sous deux tunnels insectproof localisés sur le centre Inra d'Avignon, ceci afin d'empêcher tout butinage des fleurs par les insectes (Fig. 1). Les tunnels, composés de 4 rangs de $18 \mathrm{~m}$ de long, ont été semés chacun avec deux lignées de colza d'hiver (Brassica napus L.) : la variété hybride F1 Exocet (mâle fertile; Fig. 2A), obtention Monsanto , et son parent femelle, mâle stérile (Fig. 2B). Les deux tunnels ont été semés avec trois semaines d'écart, le premier le 21 novembre 2016 et le second le 12 décembre 2016, dans le but d'avoir un décalage de floraison 

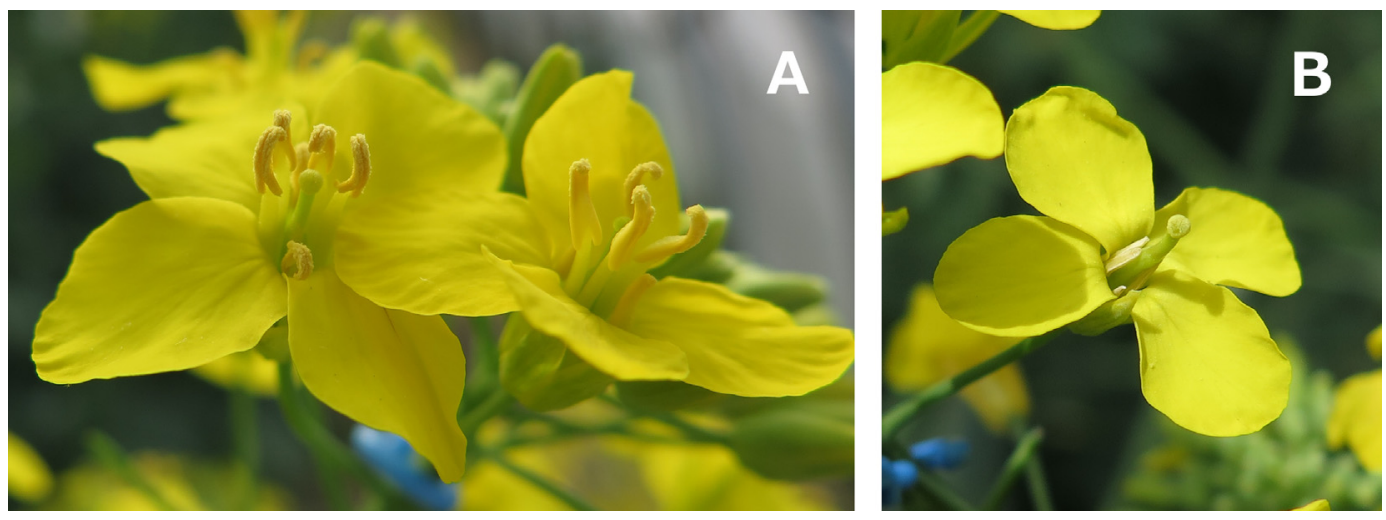

Fig. 2. (A) Fleurs mâle fertile F1 de colza d'hiver Exocet au $1^{\mathrm{er}}$ jour d'anthèse (à droite) et $2^{\mathrm{e}}$ jour d'anthèse (à gauche). (B) Fleur mâle stérile, lignée parentale d'«Exocet», au $1^{\text {er }}$ jour d'anthèse. (C) Taïna Lemoine / Inra.

et de disposer ainsi d'une floraison plus étendue dans le temps pour nos mesures. Les plantes étaient arrosées quotidiennement par un système de goutte-à-goutte afin d'éviter qu'elles ne soient en déficit hydrique. La température instantanée était enregistrée toutes les 2 min 30 par un capteur $\mathrm{HOBO}^{\circledR}$ Pro v2 (Onset ${ }^{\circledR}$ Computer Corporation, USA).

\subsection{Identification de cohortes de fleurs}

Nous avons effectué les mesures de taux de sécrétion brut de nectar à cinq dates, échelonnées entre le 14 avril et le 29 mai 2017, afin de pouvoir échantillonner le nectar sous des conditions de températures les plus variées possible tout au long de la floraison du colza (Tab. 1). Ces mesures ont été réalisées sur des fleurs de même âge, au premier jour d'anthèse, en identifiant une cohorte de fleurs pour chaque date. Pour identifier une cohorte, nous déposions la veille des relevés entre $16 \mathrm{~h} 00$ et $18 \mathrm{~h} 00$ GMT (heure locale moins deux heures, proche de l'heure solaire) un premier marqueur de fleur (Cherry; Filpack agricole, France) sur 12 hampes florales choisies au hasard par lignée et par date sur l'ensemble des plantes, sous le bouton totalement fermé positionné le plus bas sur la hampe. La floraison du colza est acropétale, c'est-à-dire qu'elle se réalise de la base de la hampe vers le sommet, mais pas de façon stricte, de sorte que l'on peut trouver des fleurs épanouies au-dessus de fleurs encore au stade bouton (Fig. 3). Nous avons donc sectionné toutes les fleurs épanouies situées au-dessus du bouton marqué en haut du pédoncule (Fig. 3), pour ne disposer que de boutons fermés au-dessus de notre marqueur. Nous déposions ensuite le jour des relevés autour de 08h00 GMT un deuxième marqueur de fleur sur ces mêmes hampes au-dessus de la fleur épanouie en position la plus haute. Nous sectionnions alors tous les boutons non épanouis situés au-dessous de ce deuxième marqueur en haut du pédoncule, de façon à ne conserver que des fleurs épanouies du matin entre les deux marqueurs. Cette élimination de fleurs pouvait avoir pour effet potentiel d'augmenter les flux de sève vers les fleurs non retirées, mais nous avons considéré ce potentiel effet comme négligeable. Nous disposions ainsi de 2 à 5 fleurs de même âge par hampe florale (Fig. 4). Pour faciliter l'organisation des mesures, les fleurs d'une même hampe recevaient le même traitement, à savoir, tout d'abord un
Tableau 1. Températures d'échantillonnage mesurées pour chaque date de relevés.

\begin{tabular}{llcl}
\hline Date & \multicolumn{3}{c}{ Température $\left({ }^{\circ} \mathrm{C}\right)$} \\
\cline { 2 - 4 } & Moyenne & Minimale & Maximale \\
\hline 14.04 .2017 & 24,98 & 22,07 & 28,66 \\
25.04 .2017 & 18,9 & 9,17 & 25,97 \\
01.05 .2017 & 17,11 & 12,13 & 22,12 \\
23.05 .2017 & 30,75 & 24,27 & 34,58 \\
29.05 .2017 & 33,43 & 27,71 & 35,74 \\
\hline
\end{tabular}

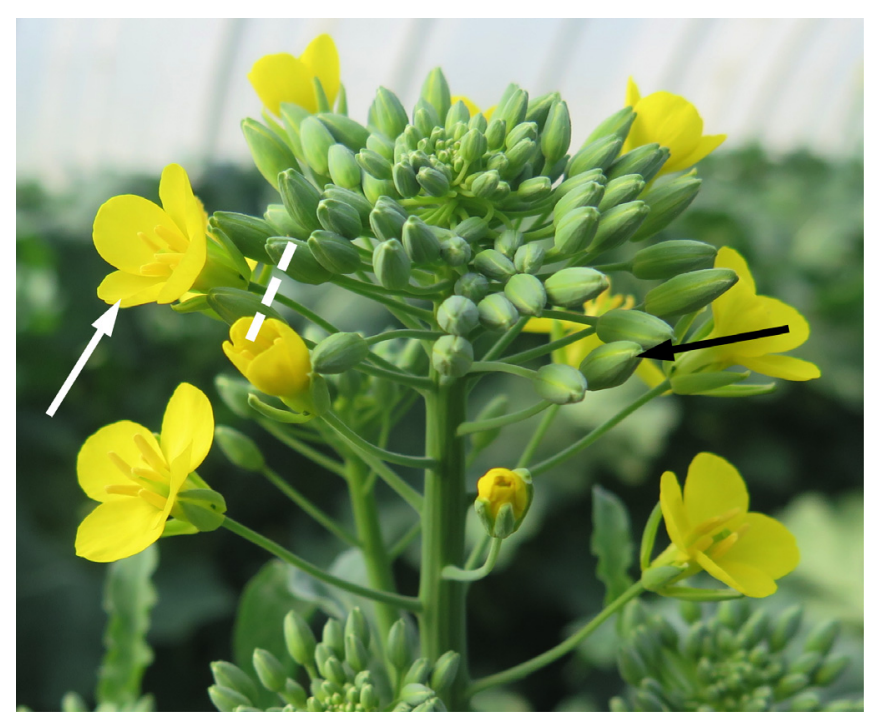

Fig. 3. Hampe florale mâle fertile de colza dont une fleur épanouie (flèche blanche à gauche) se situe au-dessus d'un bouton encore fermé (flèche noire à droite). La ligne blanche en pointillés symbolise la zone où les fleurs étaient sectionnées, en haut du pédoncule, pour disposer de fleurs du même âge dans les cohortes sélectionnées. (C) Taïna Lemoine / Inra.

prélèvement initial au même moment, puis une mesure de sécrétion nectarifère au même moment. L'heure de prélèvement initial était alors notée sur une étiquette à fil attachée autour de la hampe. 


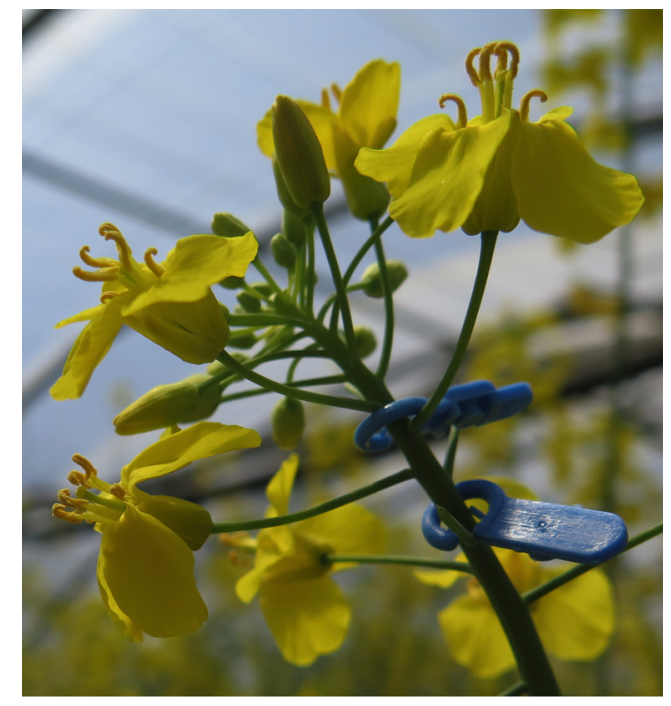

Fig. 4. Hampe florale de colza avec deux marqueurs de fleur délimitant une cohorte de deux fleurs mâle fertile de même âge. () Stan Chabert / Inra.

\subsection{Mesure du taux de sécrétion brut de nectar}

À chaque date de relevé, les prélèvements initiaux de nectar ont été réalisés successivement sur les 12 hampes de chacune des deux lignées entre $08 \mathrm{~h} 00$ et $12 \mathrm{~h} 00$ GMT. Chaque hampe florale servait ensuite pour une mesure du taux de sécrétion brut unique, avec un intervalle de temps différent testé pour chaque hampe. La gamme d'intervalles de temps testée s'échelonnait de la façon suivante: $15 \mathrm{~min}, 30 \mathrm{~min}$, $45 \mathrm{~min}, 1 \mathrm{~h}, 1 \mathrm{~h} 30,2 \mathrm{~h}, 3 \mathrm{~h}, 4 \mathrm{~h}, 5 \mathrm{~h}, 6 \mathrm{~h}, 7 \mathrm{~h}$ et $8 \mathrm{~h}-$ soit 12 intervalles de temps pour les 12 hampes. Les hampes étant constituées de 2 à 5 fleurs marquées, nous disposions ainsi de 2 à 5 répétitions de mesure par intervalle de temps par lignée et par date. La première hampe florale recevant le traitement de prélèvement initial servait pour la mesure de taux de sécrétion brut avec le plus grand intervalle de temps ( 8 heures), puis la deuxième hampe recevant le traitement de prélèvement initial servait pour la mesure de taux de sécrétion brut avec l'intervalle de temps de 7 heures, et ainsi de suite jusqu'au plus petit intervalle de temps (15 minutes). Cette organisation permettait d'éviter un trop gros décalage de temps entre les mesures, avec notamment le midi solaire inclus dans tous les intervalles de temps.

Les récoltes de nectar des prélèvements initiaux ont été réalisées à l'aide de microcapillaires de $1 \mu \mathrm{L}$, puis de $0.5 \mu \mathrm{L}$ pour retirer le nectar résiduel potentiel, cette étape ayant été réalisée avec précaution pour ne pas endommager les nectaires. Seul le nectar des deux nectaires latéraux était échantillonné dans chaque fleur (Fig. 5), ces nectaires produisant $95 \%$ du nectar sécrété par la fleur (Davis et al., 1986, 1998). Lorsque le nectar était visqueux, c'est-à-dire dans les situations de fortes concentrations et de faibles températures (Nicolson et Thornburg, 2007), situations qui intervenaient systématiquement au moins en fin d'après-midi après $16 \mathrm{~h} 00$ GMT, les microcapillaires étaient laissés en contact avec les nectaires durant 3 à 5 minutes (Fig. 6). Enfin, pour la valeur de température associée à chaque mesure de taux de sécrétion brut de nectar, nous avons calculé la moyenne de l'ensemble des températures instantanées

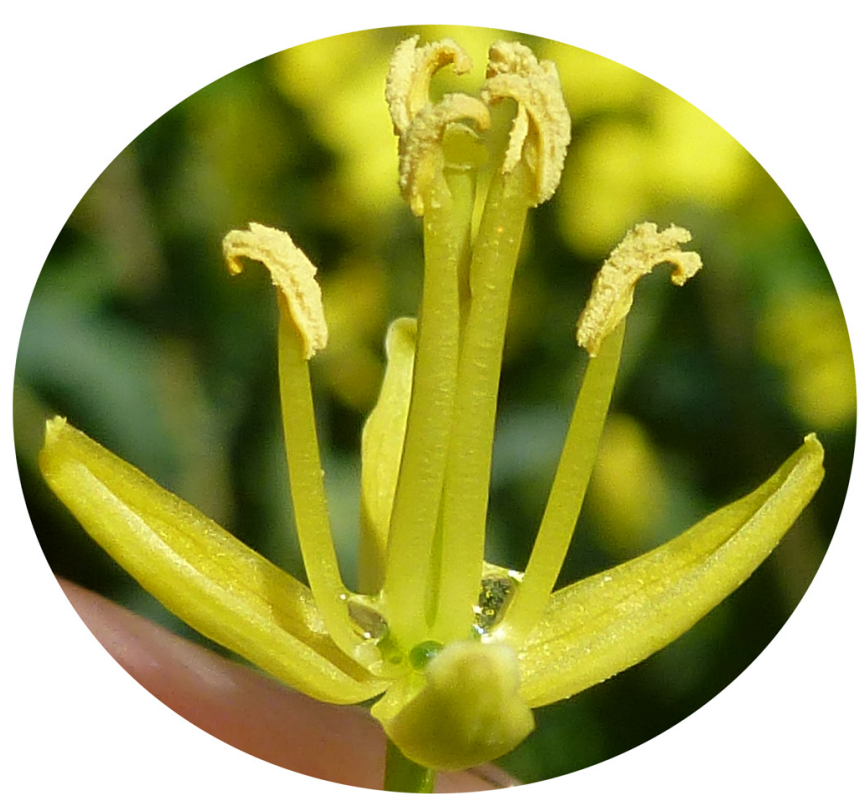

Fig. 5. Fleur mâle fertile de colza d'hiver dont on a retiré les pétales et sur laquelle on distingue les deux gouttes de nectar situées au niveau des nectaires latéraux, à la base du pistil et des étamines courtes. (C) Nicolas Cerruti / Terres Inovia.

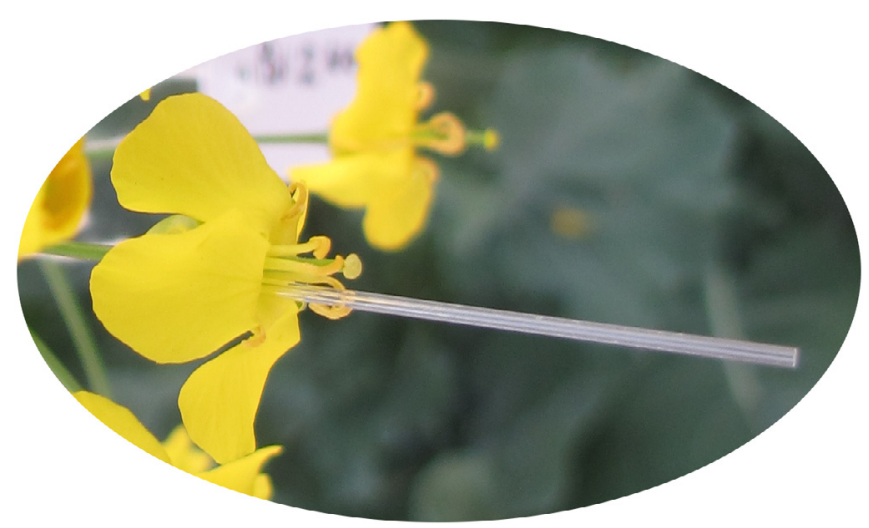

Fig. 6. Échantillonnage de nectar dans une fleur mâle fertile de colza à l'aide d'un microcapillaire. (C) Stan Chabert / Inra.

enregistrées par le capteur durant la période comprise entre l'heure (à 5 minutes près) du prélèvement initial et l'heure de la mesure du taux de sécrétion brut.

La concentration du nectar en STD a été mesurée à l'aide de deux réfractomètres adaptés pour les petits volumes, permettant une lecture jusqu'à des volumes de $0,05 \mu \mathrm{L}$, pour des gammes de 0-50 et 45-80\% Brix (Eclipse 45-81 et 45-82; Bellingham \& Stanley Ltd, UK). La masse de STD était ensuite obtenue à l'aide de la formule de conversion de Cruden et Hermann (1983) :

$$
\mathrm{M}=\mathrm{C} \cdot\left(4,6 \cdot 10^{-5} \cdot \mathrm{C}+9,946 \cdot 10^{-3}\right) . \mathrm{V},
$$

où $\mathrm{M}$ est la masse de STD en mg, V le volume de nectar en $\mu \mathrm{L}$, et $\mathrm{C}$ la concentration du nectar en $\mathrm{g}$ de STD pour $100 \mathrm{~g}$ de solution (\% Brix). 

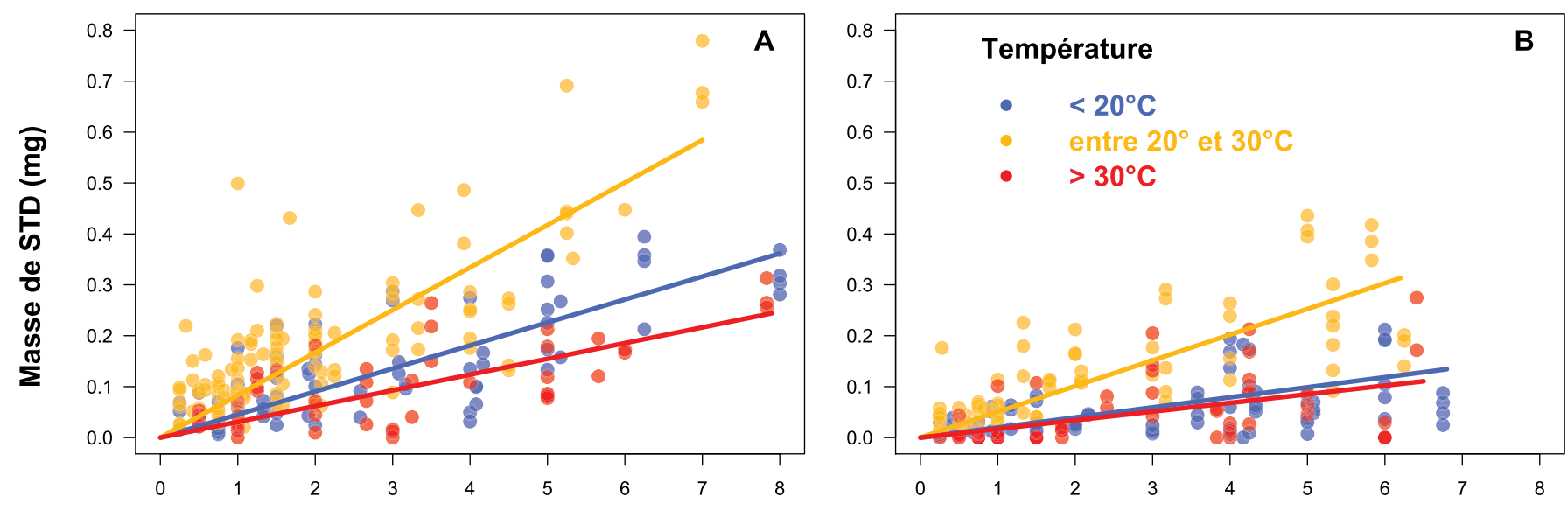

Nombre d'heures après le prélèvement initial

Fig. 7. Masse de sucres totaux dissous (STD) sécrétée par fleur pour (A) la lignée hybride F1 et (B) son parent femelle mâle stérile en fonction du nombre d'heures écoulées après le prélèvement initial et selon la catégorie de température.

Tableau 2. Vitesses de sécrétion nectarifère brutes estimées par catégorie de température pour chaque lignée.

\begin{tabular}{lllll}
\hline Lignée & $\begin{array}{l}\text { Catégorie de } \\
\text { température }\left({ }^{\circ} \mathrm{C}\right)\end{array}$ & $\begin{array}{l}\text { Nombre de fleurs } \\
\text { échantillonnées }\end{array}$ & $\begin{array}{l}\text { Vitesse de sécrétion } \\
\left(\text { ng STD . }{ }^{-1} \text { ) }\right.\end{array}$ & $\begin{array}{l}\text { Intervalle de confiance à 95\% } \\
\text { Borne } \\
\text { inférieure }\end{array}$ \\
\hline F1 & $<20$ & 73 & 45,18 & 40,60 \\
sorne \\
\end{tabular}

STD : sucres totaux dissous.

\subsection{Analyses statistiques}

Le nectar étant sécrété de façon continue (Pacini et Nepi, 2007), nous avons estimé les pentes, ainsi que les intervalles de confiance à $95 \%$ associés, de la masse de STD en fonction de la durée écoulée entre le prélèvement initial et la mesure effectuée à l'aide de modèles linéaires mécanistes de la forme $\hat{y}=\hat{a} . x$ (méthode d'inférence statistique dite des moindres carrés; Bolker, 2008), pour chacune des deux lignées (F1 et MS) et pour chaque catégorie de température $\left(<20^{\circ} \mathrm{C}\right.$; entre $20^{\circ}$ et $30^{\circ} \mathrm{C} ;>30^{\circ} \mathrm{C}$ ). Deux moyennes dont les intervalles de confiance à $95 \%$ ne se recouvrent pas ne peuvent être considérées comme statistiquement identiques, avec une chance de $5 \%$ de se tromper (Nakagawa et Cuthill, 2007). Ces analyses ont été réalisées avec le logiciel $\mathrm{R}$, version 3.2.0 (R Core Team, 2015).

\section{Résultats}

Les températures des relevés ont varié de $9{ }^{\circ} \mathrm{C}$ à $36^{\circ} \mathrm{C}$ sous les tunnels (Tab. 1). On peut tout d'abord constater que la masse de STD contenue dans les fleurs augmentait de façon constante au cours du temps après le prélèvement initial de nectar pour les deux lignées, F1 et MS, et ce dès 15 minutes et quelle que soit la température d'échantillonnage (Fig. 7). La vitesse de sécrétion, en masse de STD, est de deux à trois fois plus forte entre $20^{\circ} \mathrm{C}$ et $30^{\circ} \mathrm{C}$ qu'en-dessous de $20^{\circ} \mathrm{C}$ et qu'au-dessus de $30^{\circ} \mathrm{C}$ pour les deux lignées (les intervalles de confiance à $95 \%$ ne se chevauchaient pas ; Tab. 2). D'autre part, cette vitesse de sécrétion était moindre pour la lignée MS par rapport à la lignée $\mathrm{F} 1$, et ce pour toutes les plages de température (Tab. 2). La vitesse de sécrétion de la lignée MS équivalait à $61 \%$ de celle de la lignée F1 entre $20^{\circ} \mathrm{C}$ et $30^{\circ} \mathrm{C}$. En-dessous de $20^{\circ} \mathrm{C}$, cette vitesse équivalait à $35 \%$ de celle de la lignée $\mathrm{F} 1$, et au-dessus de $30^{\circ} \mathrm{C}$ à $55 \%$ de celle de la lignée F1.

\section{Discussion}

\subsection{L'effet unimodal de la température sur la sécrétion nectarifère}

Pacini et al. (2003) distinguent deux types de nectaires floraux: ceux dont l'amidon, qui sert de réactif pour la production de sucres, provient directement de la photosynthèse et qui sécrètent par conséquent du nectar en continu et en petites quantités lorsque la photosynthèse est active, et ceux 
dont l'amidon est préalablement stocké dans leur parenchyme avant sécrétion, et qui peuvent sécréter de grandes quantités de nectar en très peu de temps indépendamment de la photosynthèse (ces nectaires peuvent donc par exemple sécréter la nuit dans le cas de pollinisateurs nocturnes). Le colza, comme l'ensemble des Brassicaceae, appartient au premier type de plante où il n'y a pas de stockage d'amidon (Davis et al., 1986, 1998). Or, l'activité photosynthétique des plantes admet un optimum de température, cet optimum étant lui-même dépendant de la température à laquelle les plantes se sont développées. Les plantes ont ainsi une capacité d'acclimatation qui leur permet d'adapter leur activité photosynthétique au climat rencontré (Berry et Björkman, 1980; Yamori et al., 2014). Les variations de sécrétion nectarifère dues à la température s'expliqueraient donc directement par l'effet unimodal de la température sur la photosynthèse. De plus, l'optimum thermique observé pour la sécrétion de nectar pourrait également varier, à l'instar de la photosynthèse, suivant la température de développement des plantes: ceci a en effet déjà été observé par Huber (1956).

C'est à notre connaissance la troisième fois qu'un tel effet de la température a été mis en évidence sur une vitesse de sécrétion nectarifère (Fig. 7 et Tab. 2; Findlay et al., 1971; Nicolson, 1995), confirmant ce qui a déjà été observé chez plusieurs espèces sur le taux de sécrétion apparent (Huber, 1956; Villarreal et Freeman, 1990; Petanidou et Smets, 1996; Pacini et Nepi, 2007; Petanidou, 2007; Takkis et al., 2015).

\subsection{La lignée MS sécrète deux fois moins vite que la lignée F1}

La lignée MS sécrétait du nectar en moyenne deux fois moins vite que la lignée F1 sur l'ensemble des températures (Fig. 7 et Tab. 2). Ces résultats rejoignent en partie les observations de Mesquida et al. (1991) et de Pierre et al. (1999) réalisées sur les volumes de nectar sécrétés par des lignées pures MF et MS avec la mesure du taux de sécrétion apparent. Cette différence de vitesse de sécrétion peut s'expliquer en partie par la mise en place du système de stérilité mâle cytoplasmique: lors de la conception de ce système, les premières lignées MS obtenues en combinant le génome nucléaire du colza avec le cytoplasme du radis Raphanus sativus ont eu pour effet d'altérer le développement des nectaires (Pelletier et Budar, 2015).

La différence de vitesse de sécrétion demeurant encore aujourd'hui entre les deux types de lignées peut également provenir de la différence de taille entre les fleurs, et donc de taille entre les nectaires. Il a en effet été montré chez plusieurs espèces que la quantité de nectar sécrétée est corrélée positivement avec la taille des nectaires (Teuber et al., 1980; Dafni et al., 1988; Petanidou et al., 2000; Galetto et Bernardello, 2004). C'est l'idée qui a été proposée par Sammataro et al. (1985) pour expliquer les différences d'attractivité entre variétés chez le tournesol. Il serait donc intéressant de mesurer la taille des nectaires ou des fleurs pour tester si cette différence de vitesse de sécrétion observée entre les deux lignées provient effectivement de cette différence de taille ou non.

\subsection{Une méthode rigoureuse pour comparer la sécrétion nectarifère de différentes lignées ou variétés}

Des lignées MS plus productives en nectar pourraient être sélectionnées (Mesquida et al., 1991; Pierre et al., 1999), à l'instar des lignées MF conventionnelles (Kamler, 1984; Davis, 2001). Un certain niveau d'héritabilité pour le caractère de sécrétion de nectar a en effet été mis en évidence chez plusieurs espèces (Mitchell, 2004 ; Leiss et Klinkhamer, 2005 ; Kaczorowski et al., 2008). Tout en sachant que la quantité de nectar sécrétée n'est pas le seul critère pour caractériser l'attractivité d'une espèce ou d'une lignée : l'accessibilité du nectar, comme la longueur des fleurons chez le tournesol (Mallinger et Prasifka, 2017), et la composition en sucres du nectar (Nicolson, 2007) y contribuent également.

Pour envisager un tel programme de sélection, la méthode que nous avons utilisée qui mesure le taux de sécrétion brut de nectar en STD sur des fleurs au $1^{\mathrm{er}}$ jour d'anthèse peut être retenue, en veillant à échantillonner le nectar des différentes lignées dans des conditions environnementales similaires, en particulier pour la température. L'intervalle de temps à retenir entre le prélèvement initial de nectar et la mesure peut être de 6 ou 8 heures, avec un prélèvement initial qui peut s'effectuer le matin entre $09 \mathrm{~h} 00$ et $12 \mathrm{~h} 00$, heure locale, et une mesure qui peut s'effectuer jusqu'à $18 \mathrm{~h} 00$. Ensuite, le nectar devient très visqueux et monte très lentement dans les microcapillaires. La sécrétion nectarifère est en effet bien constante sur un pas de temps de 6-8 heures quelle que soit la température (Fig. 7). Cette sécrétion étant directement dépendante de la photosynthèse, il est impératif que le prélèvement initial de nectar ait lieu lorsqu'il y a photosynthèse, c'est-à-dire durant le jour à partir du moment où le rayonnement est suffisant. Au-delà d'un intervalle de temps de 8 heures entre le prélèvement initial et la mesure, le processus de sécrétion devrait donc a priori continuer, jusqu'au moment où le rayonnement devient limitant pour la photosynthèse. Enfin, le prélèvement initial de nectar peut être réalisé à l'aide de papier Whatman, à la place de microcapillaires, afin d'être certain de ne pas endommager les nectaires (McKenna et Thomson, 1988).

\subsection{Perspectives: quels autres facteurs prendre en compte?}

\subsection{1 Âge de la fleur et pollinisation}

D'autres facteurs sont encore à prendre en compte pour expliquer la variabilité de la sécrétion nectarifère, avec en premier lieu l'âge de la fleur, la sécrétion se réalisant en continu jusqu'au moment de sa sénescence où le nectar est peu à peu réabsorbé (Nepi et Stpiczyńska, 2008). C'est un facteur particulièrement important pour les espèces protogynes ou protandres, comme le tournesol ou la carotte, où la sécrétion peut varier selon la phase sexuelle qui évolue avec l'âge (Langenberger et Davis, 2002; Wist et Davis, 2006, 2008; Varga et al., 2013). Il faut également tenir compte de l'effet de la pollinisation, qui peut déclencher précocement le processus de sénescence de la fleur et du coup interrompre la sécrétion (Stead, 1992; van Doorn, 1997; Rogers, 2006). Avec ces connaissances, nous pourrions ainsi estimer de façon précise la quantité totale de sucres produite par un hectare de culture 
d'une espèce et d'une lignée donnée, disponible pour les abeilles : de premières approximations ont été proposées par Pierre et Emeillat (2009) et Thom et al. (2016). Et à terme identifier une charge de colonies d'abeilles mellifères par unité de surface à ne pas dépasser si l'on souhaite éviter que les colonies ne rentrent trop en compétition les unes avec les autres pour le nectar.

\subsubsection{Humidité du sol}

Enfin la teneur en eau du sol apparaît comme un dernier facteur important pour la sécrétion nectarifère. Différentes études sur espèces sauvages ont montré que la sécrétion de nectar augmente avec l'humidité du sol (Villarreal et Freeman, 1990 ; Wyatt et al., 1992 ; Carroll et al., 2001 ; Waser et Price, 2016; Gallagher et Campbell, 2017). Gillespie et al. (2015) ont observé une relation similaire en production de semence hybride d'oignon, mais avec un optimum d'humidité au-delà duquel la sécrétion diminue. Ceci rejoint le fait que l'activité photosynthétique des plantes diminue en situation de déficit hydrique (Chaves et al., 2009), comme en situation d'excès hydrique (Balakhnina, 2015). Leiss et Klinkhamer (2005) ont quant à eux montré, à l'instar de Boose (1997), l'existence d'une interaction génotype-environnement sur la sécrétion nectarifère : l'effet positif de l'humidité du sol sur la sécrétion chez la vipérine commune (Echium vulgare) s'observe surtout pour des génotypes peu nectarifères avec un système racinaire peu développé. À l'inverse, des génotypes très nectarifères avec un système racinaire bien développé ont une forte capacité de sécrétion en condition de sol sec comme en condition de sol humide. Il apparaît ainsi que des différences de sécrétion nectarifère entre lignées ou entre variétés peuvent ne se révéler que dans certains contextes environnementaux, par exemple lorsque les plantes sont en condition de stress. Lindström et al. (2017) viennent par exemple de montrer que l'irrigation permet de compenser l'effet négatif des méligèthes sur la sécrétion nectarifère du colza (Kirk et al., 1995; Krupnick et al., 1999).

\section{Lexique}

Anémophilie qualifie une espèce végétale dont la pollinisation, et par extension la fécondation, dépend en majorité du vent. Deux procédés peuvent intervenir: la dispersion du pollen atmosphérique, ou le secouage des fleurs dans le cas de plantes hermaphrodites

Entomophilie qualifie une espèce végétale dont la pollinisation, et par extension la fécondation, dépend en majorité de l'activité des insectes

Protandre fleur hermaphrodite dont la phase mâle précède la phase femelle

Protogyne fleur hermaphrodite dont la phase femelle précède la phase mâle

Remerciements. Nous tenons à remercier l'ensemble des membres de l'Installation Expérimentale de l'unité EMMAH du centre INRA PACA qui nous ont permis de disposer d'une très belle culture de colza sous tunnel: Gilles Continsouzas, Véronique Desfonds, Serge Reno, Jérémy Theodore et
Frédéric Venault. Nous remercions également la société Monsanto ${ }^{(8)}$ qui nous a gracieusement mis ses semences à disposition pour réaliser cette étude. Nous remercions enfin Isabelle Dajoz et une/un autre relectrice/teur anonyme pour leur relecture approfondie qui a permis d'améliorer grandement la qualité de cet article.

Conflits d'intérêts. Les auteurs déclarent n'avoir aucun lien d'intérêt concernant les données publiées dans cet article.

\section{Références}

Anderson GJ, Bernardello G, Lopez PS, Crawford DJ, Stuessy TF. 2000a. Reproductive biology of Wahlenbergia (Campanulaceae) endemic to Robinson Crusoe Island (Chile). Pl Syst Evol 223(1): 109-123.

Anderson GJ, Bernardello G, Lopez P, Stuessy TF, Crawford DJ. 2000b. Dioecy and wind pollination in Pernettya rigida (Ericaceae) of the Juan Fernández Islands. Bot J Linn Soc 132 (2): $121-141$.

Anderson GJ, Bernardello G, Stuessy TF, Crawford DJ. 2001. Breeding system and pollination of selected plants endemic to Juan Fernández Islands. Amer J Bot 88(2): 220-233.

Balakhnina TI. 2015. Plant responses to soil flooding. In: Tripathi BN, Müller M, eds. Stress responses in plants: mechanisms of toxicity and tolerance. Springer International Publishing, pp. 115-142.

Baude M, Kunin WE, Boatman ND, et al. 2016. Historical nectar assessment reveals the fall and rise of floral resources in Britain. Nature 30(7588): 85-88.

Bernardello G. 2007. A systematic survey of floral nectaries. In: Nicolson SW, Nepi M, Pacini E, eds. Nectaries and nectar. Springer, pp. 19-128.

Bernardello G, Galetto L, Anderson GJ. 2000. Floral nectary structure and nectar chemical composition of some species from Robinson Crusoe Island (Chile). Can J Bot 78(7): 862-871.

Bernardello G, Anderson GJ, Stuessy TF, Crawford DJ. 2001. A survey of floral traits, breeding systems, floral visitors, and pollination systems of the angiosperms of the Juan Fernández Islands (Chile). Bot Rev 67(3): 255-308.

Berry J, Björkman O. 1980. Photosynthetic response and adaptation to temperature in higher plants. Annual Rev PlPhysiol31(1): 491-543.

Bolker BM. 2008. Ecological models and data in R. Princeton University Press.

Boose DL. 1997. Sources of variation in floral nectar production rate in Epilobium canum (Onagraceae): implications for natural selection. Oecologia 110(4): 493-500.

Bosi G. 1973. Méthode rapide pour la détermination par chromatographie en phase gazeuse des glucides du nectar: technique de prélèvement du nectar et de préparation des éthers triméthylsilyles en présence d'eau. Apidologie 4(1): 57-64.

Branstetter MG, Danforth BN, Pitts JP, et al. 2017. Phylogenomic insights into the evolution of stinging wasps and the origins of ants and bees. Curr Biol 27(7): 1019-1025.

Búrquez A, Corbet SA. 1991. Do flowers reabsorb nectar? Funct Ecol 5(3): 369-379.

Cappellari SC, Schaefer H, Davis CC. 2013. Evolution: pollen or pollinators - Which came first? Curr Biol 23(8): R316-R318.

Carroll AB, Pallardy SG, Galen C. 2001. Drought stress, plant water status, and floral trait expression in fireweed, Epilobium angustifolium (Onagraceae). Amer J Bot 88(3): 438-446.

Chaves MM, Flexas J, Pinheiro C. 2009. Photosynthesis under drought and salt stress: regulation mechanisms from whole plant to cell. Ann Bot (Oxford) 103(4): 551-560. 
Corbet SA. 2003. Nectar sugar content: estimating standing crop and secretion rate in the field. Apidologie 34(1): 1-10.

Cruden RW, Hermann SM. 1983. Studying nectar? Some observations on the art. In: Bentley B, Elias T, eds. The biology of nectaries. New York: Columbia University Press, pp. 223-241.

Cruden RW, Hermann SM, Peterson S. 1983. Patterns of nectar production and plant-pollinator coevolution. In: Bentley B, Elias T, eds. The biology of nectaries. New York: Columbia University Press, pp. 223-241.

Dafni H, Lensky Y, Fahn A. 1988. Flower and nectar characteristics of nine species of Labiatae and their influence on honeybee visits. $J$ Apic Res 27(2): 103-114.

Davis AR. 2001. Searching and breeding for structural features of flowers correlated with high nectar-carbohydrate production. Acta Hortic 561: 107-121.

Davis AR, Peterson RL, Shuel RW. 1986. Anatomy and vasculature of the floral nectaries of Brassica napus (Brassicaceae). Can J Bot 64 (11): 2508-2516.

Davis AR, Pylatuik JD, Paradis JC, Low NH. 1998. Nectarcarbohydrate production and composition vary in relation to nectary anatomy and location within individual flowers of several species of Brassicaceae. Planta 205(2): 305-318.

Findlay N, Reed ML, Mercer FV. 1971. Nectar production in Abutilon III. Sugar secretion. Austral J Biol Sci 24(3): 665-675.

Friis EM, Crane PR, Pedersen KR. 2011. History and evolution of pollination in angiosperms. In: Early flowers and angiosperm evolution. Cambridge University Press, pp. 415-443.

Galetto L, Bernardello G. 2004. Floral nectaries, nectar production dynamics and chemical composition in six Ipomoea species (Convolvulaceae) in relation to pollinators. Ann Bot (Oxford) 94 (2): 269-280.

Gallagher MK, Campbell DR. 2017. Shifts in water availability mediate plant-pollinator interactions. New Phytol 215(2): 792802.

Garibaldi LA, Requier F, Rollin O, Andersson GK. 2017. Towards an integrated species and habitat management of crop pollination. Curr Opin Insect Sci 21: 105-114.

Gillespie S, Long R, Williams N. 2015. Indirect effects of field management on pollination service and seed set in hybrid onion seed production. J Econ Entomol 108(6): 2511-2517.

Huber H. 1956. Die Abhängigkeit der Nektarsekretion von Temperatur, Luft- und Bodenfeuchtigkeit. Planta 48(1): 47-98.

Kaczorowski RL, Juenger TE, Holtsford TP. 2008. Heritability and correlation structure of nectar and floral morphology traits in Nicotiana alata. Evolution 62(7): 1738-1750.

Kamler F. 1984. Selection of lines of winter rape (Brassica napus v. arvensis) for nectar production. $V^{e}$ Symposium International sur la Pollinisation. 27-30 Septembre 1983, Versailles. Les Colloques de l'Inra 21: 135-139.

Kearns CA, Inouye DW. 1993. Techniques for pollination biologists. University Press of Colorado.

Kirk WD, Ali M, Breadmore KN. 1995. The effects of pollen beetles on the foraging behaviour of honey bees. J Apic Res 34(1): 15-22.

Krupnick GA, Weis AE, Campbell DR. 1999. The consequences of floral herbivory for pollinator service to Isomeris arborea. Ecology 80(1): 125-134.

Langenberger MW, Davis AR. 2002. Temporal changes in floral nectar production, reabsorption, and composition associated with dichogamy in annual caraway (Carum carvi; Apiaceae). Amer J Bot 89(10): 1588-1598.

Leiss KA, Klinkhamer PGL. 2005. Genotype by environment interactions in the nectar production of Echium vulgare. Funct Ecol 19(3): 454-459.
Lindström SAM, Klatt BK, Smith HG, Bommarco R. 2017. Crop management affects pollinator attractiveness and visitation in oilseed rape. Basic Appl Ecol in press.

Luo EY, Ogilvie JE, Thomson JD. 2014. Stimulation of flower nectar replenishment by removal: a survey of eleven animal-pollinated plant species. J Pollinat Ecol 12(7): 52-62.

Mallick SA. 2000. Technique for washing nectar from the flowers of Tasmanian leatherwood (Eucryphia lucida Eucryphiaceae). Austral Ecol 25(2): 210-212.

Mallinger RE, Prasifka JR. 2017. Bee visitation rates to cultivated sunflowers increase with the amount and accessibility of nectar sugars. J Appl Entomol 141(7): 561-573.

McKenna MA, Thomson JD. 1988. A technique for sampling and measuring small amounts of floral nectar. Ecology 69(4): 13061307.

Mesquida J, Marilleau R, Pham-Delègue MH, Renard M. 1988. A study of rapeseed (Brassica napus L. var. oleifera Metzger) flower nectar secretions. Apidologie 19(3): 307-318.

Mesquida J, Pham-Delègue MH, Marilleau R, Le Métayer M, Renard M. 1991. La sécrétion nectarifère des fleurs de cybrides mâlesstériles de colza d'hiver (Brassica napus L). Agronomie 11(3): 217-227.

Michez D, Vanderplanck M, Engel MS. 2012. Fossil bees and their plant associates. In: Patiny S, ed. Evolution of plant-pollinator relationships. Cambridge University Press, pp. 103-164.

Mitchell RJ. 2004. Heritability of nectar traits: why do we know so little? Ecology 85(6): 1527-1533.

Morrant DS, Schumann R, Petit S. 2009. Field methods for sampling and storing nectar from flowers with low nectar volumes. Ann Bot 103(3): 533-542.

Nakagawa S, Cuthill IC. 2007. Effect size, confidence interval and statistical significance: a practical guide for biologists. Biol Rev 82(4): 591-605.

Nepi M, Stpiczyńska M. 2008. The complexity of nectar: secretion and resorption dynamically regulate nectar features. Naturwissenschaften 95(3): 177-184.

Nicolson SW. 1995. Direct demonstration of nectar reabsorption in the flowers of Grevillea robusta (Proteaceae). Funct Ecol 9(4): 584-588.

Nicolson SW. 2007. Nectar consumers. In: Nicolson SW, Nepi M, Pacini E, eds. Nectaries and nectar. Springer, pp. 289-342.

Nicolson SW, Thornburg RW. 2007. Nectar chemistry. In: Nicolson SW, Nepi M, Pacini E, eds. Nectaries and nectar. Springer, pp. 215-264.

Pacini E, Nepi M. 2007. Nectar production and presentation. In: Nicolson SW, Nepi M, Pacini E, eds. Nectaries and nectar. Springer, pp. 167-214.

Pacini E, Nepi M, Vesprini JL. 2003. Nectar biodiversity: a short review. Pl Syst Evol 238(1): 7-21.

Pelletier G, Budar F. 2015. Brassica Ogu-INRA cytoplasmic male sterility: an example of successful plant somatic fusion for hybrid seed production. In: Li X-Q, Donnelly DJ, Jensen TG, eds. Somatic genome manipulation: advances, methods and applications. New York: Springer, pp. 199-216.

Percival MS. 1961. Types of nectar in angiosperms. New Phytol 60 (3): 235-281.

Petanidou T. 2007. Ecological and evolutionary aspects of floral nectars in Mediterranean habitats. In: Nicolson SW, Nepi M, Pacini E, eds. Nectaries and nectar. Springer, pp. 343-375.

Petanidou T, Goethals V, Smets E. 2000. Nectary structure of Labiatae in relation to their nectar secretion and characteristics in a Mediterranean shrub community - does flowering time matter? Pl Syst Evol 225(1): 103-118. 
Petanidou T, Smets E. 1996. Does temperature stress induce nectar secretion in Mediterranean plants? New Phytol 133(3): 513-518.

Petit S, Rubbo N, Schumann R. 2011. Nectar collected with microcapillary tubes is less concentrated than total nectar in flowers with small nectar volumes. Austral J Bot 59(6): 593-599.

Pierre J, Emeillat R. 2009. Les variétés de colza actuelles sont-elles peu nectarifères pour les abeilles? Bull Tech Apic 36(1): $17-24$.

Pierre J, Renard M. 2010. Bilan de 30 ans de travaux de recherche effectués en France sur la pollinisation du colza. OCL 17(3): 121-126.

Pierre J, Mesquida J, Marilleau R, Pham-Delègue MH, Renard M. 1999. Nectar secretion in winter oilseed rape, Brassica napus quantitative and qualitative variability among 71 genotypes. Plant Breed 118(6): 471-476.

Poinar GO, Danforth BN. 2006. A fossil bee from Early Cretaceaous Burmese amber. Science 314(5799): 614

Prys-Jones OE, Corbet SA. 2011. Bumblebees. Naturalists' handbooks 6, ecology and identification, 3rd ed. Pelagic publishing.

R Core Team. 2015. R: A language and environment for statistical computing. R Foundation for Statistical Computing, Vienna, Austria. URL http://www.R-project.org/.

Rogers HJ. 2006. Programmed cell death in floral organs: how and why do flowers die? Ann Bot 97(3): 309-315.

Sammataro D, Erickson EH, Garment MB. 1985. Ultrastructure of the sunflower nectary. J Apic Res 24(3): 150-160.

Stead AD. 1992. Pollination-induced flower senescence: a review. Plant Growth Regul 11(1): 13-20.

Takhtajan AL. 1980. Outline of the classification of flowering plants (Magnoliophyta). Bot Rev 46(3): 225-359.

Takkis K, Tscheulin T, Tsalkatis P, Petanidou T. 2015. Climate change reduces nectar secretion in two common Mediterranean plants. AoB Plants 7: plv111.
Teuber LR, Albertsen MC, Barnes DK, Heichel GH. 1980. Structure of floral nectaries of alfalfa (Medicago sativa L.) in relation to nectar production. Amer J Bot 67(4): 433-439.

Thom MD, Eberle CA, Forcella F, Gesch R, Weyers S, Lundgren JG. 2016. Nectar production in oilseeds: food for pollinators in an agricultural landscape. Crop Sci 56(2): 727-739.

Vaissière BE. 1991. Honey bees, Apis mellifera L. (Hymenoptera: Apidae), as pollinators of upland cotton, Gossypium hirsutum L. (Malvaceae), for hybrid seed production. PhD, Texas A\&M University.

van Doorn WG. 1997. Effects of pollination on floral attraction and longevity. J Exp Bot 48(9): 1615-1622.

Varga S, Nuortila C, Kytöviita M-M. 2013. Nectar sugar production across floral phases in the gynodioecious protandrous plant Geranium sylvaticum. PLoS ONE 8(4): e62575.

Villarreal AG, Freeman CE. 1990. Effects of temperature and water stress on some floral nectar characteristics in Ipomopsis longiflora (Polemoniaceae) under controlled conditions. Bot Gaz 151(1): 5-9.

Waser NM, Price MV. 2016. Drought, pollen and nectar availability, and pollination success. Ecology 97(6): 1400-1409.

Wist TJ, Davis AR. 2006. Floral nectar production and nectary anatomy and ultrastructure of Echinacea purpurea (Asteraceae). Ann Bot (Oxford) 97(2): 177-193.

Wist TJ, Davis AR. 2008. Floral structure and dynamics of nectar production in Echinacea pallida var. angustifolia (Asteraceae). Int J Plant Sci 169(6): 708-722.

Wyatt R, Broyles SB, Derda GS. 1992. Environmental influences on nectar production in milkweeds (Asclepias syriaca and $A$. exaltata). Amer J Bot 79(6): 636-642.

Yamori W, Hikosaka K, Way DA. 2014. Temperature response of photosynthesis in $\mathrm{C}_{3}, \mathrm{C}_{4}$, and CAM plants: temperature acclimation and temperature adaptation. Photosynth Res 119 (1-2): 101-117.

Citation de l'article : Chabert S, Lemoine T, Fronteau L, Vaissière BE. 2017. Mesurer la sécrétion nectarifère : exemple d'une lignée hybride F1 et de son parent mâle stérile chez le colza d'hiver (Brassica napus L.). OCL 24(6): D602. 